Voix et Images

volxetimages

\title{
Aux origines du théâtre de Daniel Danis
}

Mythes de destruction et de création, rites et magie

At the Origins of Daniel Danis's Drama

Myths of Destruction and Creation, Rites and Magic

\section{En los orígenes del teatro de Daniel Danis}

Mitos de destrucción y creación, ritos y magia

\section{Hervé Guay}

Volume 40, numéro 1 (118), automne 2014

Daniel Danis

URI : https://id.erudit.org/iderudit/1028020ar

DOI : https://doi.org/10.7202/1028020ar

Aller au sommaire du numéro

\section{Éditeur(s)}

Université du Québec à Montréal

\section{ISSN}

0318-9201 (imprimé)

1705-933X (numérique)

Découvrir la revue

\section{Citer cet article}

Guay, H. (2014). Aux origines du théâtre de Daniel Danis : mythes de destruction et de création, rites et magie. Voix et Images, 40(1), 45-55. https://doi.org/10.7202/1028020ar

\section{Résumé de l'article}

Dans cet article, l'auteur s'intéresse au caractère syncrétique des mythes que convoque Daniel Danis dans plusieurs pièces et qui puisent à plusieurs sources : entre autres, la Bible et le christianisme, l'hindouisme, le judaïsme et la spiritualité amérindienne. Au-delà de ce foisonnement, l'analyse discerne dans cette dramaturgie deux grandes structures mythiques, la création et la destruction, dont l'interaction dynamise les relations entre les groupes et entre les personnages. Ces mythes, fondateurs si l'on veut, sont à leur tour inséparables de la ritualisation de la fable où se manifestent quantité de rituels et plusieurs recours à la magie, voire au surnaturel. 


\title{
AUX ORIGINES DU THEÂTRE DE DANIEL DANIS
}

\author{
Mythes de destruction et de création, rites et magie$$
+++
$$ \\ HERVÉ GUAY \\ Université du Québec à Trois-Rivières
}

One of the important insights of modern literary theory has been that every new work may also be seen as a new assemblage of material from old works ${ }^{1}$.

De tous les auteurs dramatiques québécois actuels, Daniel Danis est sans doute celui dont les pièces s'enracinent le plus profondément dans un passé à la fois lointain et familier. Non qu'elles soient toutes situées dans des temps immémoriaux - c'est même plutôt l'exception que la règle - , mais l'auteur cherche souvent à travers elles à remonter le cours d'une parole originelle. Il se plaît aussi à recourir à des formes archaïques tout en renouant, par le biais de maintes situations et de certains personnages, avec un animisme proche des sociétés préscientifiques. Ces emprunts aux structures mythiques, la récurrence des rites auxquels s'adonnent ses héros et le substrat magique qui paraît irriguer une partie de leurs pensées et de leurs comportements forment un assemblage singulier ${ }^{2}$. Je me propose pour ma part de distinguer

1 Marvin Carlson, The Haunted Stage. The Theatre as Memory Machine, Ann Arbor, University of Michigan Press, 2001, p. 4. «L'une des découvertes les plus pénétrantes de la théorie littéraire contemporaine a certainement été que toute nouvelle œuvre peut aussi être vue comme l'assemblage de matériaux issus d'œuvres plus anciennes.» Je traduis.

2 D'ailleurs, les commentateurs du thêâtre de Daniel Danis en soulignent presque toujours le caractère mythique, rituel, voire sacré. Marie-Christine Lesage remarque ainsi que Cendres de cailloux est peuplé de «jeunes fauves [...] qui organisent des rituels macabres» ("Archipels de mémoire. L'œuvre de Daniel Danis», Jeu. Revue de théâtre, nº 78, mars 1996, p. 81). À la création de la même pièce, en novembre 1993, Robert Lévesque écrivait: "Ce qui donne à l'entreprise un caractère sacré, c'est la maîtrise d'écriture de Daniel Danis, sa poésie brutale, ses images déchirées par lesquelles on devine, palpables, les misères et les secrets de personnages marqués à l'âme d'une fatalité morbide.» ("Un mauvais quart d'heure sur la planète», Le Devoir, 22 novembre 1993, p. B8.) À propos du Chant du Dire-Dire, Nadine Desrochers affirme que la voix des trois frères «est en quelque sorte la caractérisation de la parole originelle» («Le récit dans le théâtre de Daniel Danis», L'Annuaire thêâtral, n² 26, automne 1999, p. 129). La même pièce fait dire à Ève Dumas que «cette langue part des réalités les plus concrètes de la vie [...] pour s'élever vers un monde d'abstraction quasi mythologique» ("Le chant du redire», La Presse, 4 mars 2002, p. C6). Le langue-à-langue des chiens de roche amène Luc Boulanger à affirmer que «[le] texte a de forts accents de tragédie grecque» («Le langue-àlangue des chiens de roche», Voir, 18 janvier 2001, p. 49). Marie Labrecque parle d'e. Roman-dit comme du «récit initiatique de J'il, désigné comme le sauveur de son peuple après une naissance aux accents mythologiques» («Êpopée langagière», Le Devoir, 3 juin 2005, p. B2). De son côté, Jean Saint-Hilaire qualifie de «cérémonial tout de même empreint de solennité triste» la mise en scène de Terre océane orchestrée par Gill 
quelques-uns des éléments mythiques, rituels et magiques convoqués par l'auteur pour construire son œuvre et d'en faire l'analyse afin de saisir ce qu'un tel retour aux sources apporte à ce théâtre.

\section{L'ANCRAGE MYTHIQUE ET RITUEL}

Si quelques pièces de Danis renvoient à des mythes spécifiques - on pense à J'il et aux Azzédiens d'e. Roman-dit ${ }^{3}$, qui évoquent immanquablement l'épopée akkadienne de Gilgameš ${ }^{4}$-, l'auteur dramatique entretient, pour l'essentiel, ce que j'appellerais un rapport foisonnant aux mythes. Il est aisé de trouver dans son thêatre des traces de mythèmes et de rituels proches du judaïsme, du christianisme, des spiritualités autochtones ainsi que des mythologies antiques et orientales. Or, non seulement Danis fait-il des emprunts à de multiples systèmes de croyances, mais il n'hésite pas à les entremêler ${ }^{5}$, procédant ainsi à un syncrétisme religieux tout à fait personnel. e. Roman-dit en est un bon exemple. Danis y juxtapose des structures mythiques et rituelles issues, entre autres, de l'hindouisme, des spiritualités autochtones et du judaïsme. Ainsi, le «Tableau J» rappelle l'épisode du Mahâbhârata dans lequel un roi

Champagne («Un cérémonial coloré et d'une magnifique unité», Le Soleil, 28 janvier 2008, p. A2). En outre, David Blonde, dans son étude sur la violence dans le théâtre québécois, accorde une place importante à deux pièces de Danis dont il met en relief la dimension «archaïque» («Entre Oreste et Barbe-Bleue: la violence dans la scène familiale québécoise, 1981-2002», L'Annuaire théâtral, n 32, automne 2002, p. 139). Pendant ce temps, Gilbert David précise que Danis «inscrit la parole dans un cadre cérémoniel plutôt que réaliste» («Le langue-à-langue de Daniel Danis. Une parole au corps à corps», Études françaises, vol. XLIII, n 1, mai 2007, p. 71). Et enfin, Christian Saint-Pierre conclut que «[1]a dramaturgie de Danis offre une vision perçante de la condition humaine, parce qu'elle donne à voir le cycle immuable de la vie et de la mort» ("Quand la mort danse avec la vie. Cendres de cailloux de Daniel Danis», Jeu. Revue de théâtre, n 97, décembre 2000, p. 67).

3 Daniel Danis, e. Roman-dit, Paris, L'Arche, coll. «Scène ouverte», 2005, 118 p. Désormais, les références à cet ouvrage seront indiquées par le sigle $E$ suivi du folio, et placées entre parenthèses dans le texte.

4 J'il peut être vu comme une sorte de diminutif du prénom du héros assyrien, tandis que le peuple de J'il, les Azzédiens, font tout de suite penser aux Akkadiens. La langue de ces derniers est celle dans laquelle a été composée l'épopée de Gilgameš. Les assyriologues distinguent généralement la version ancienne de la version ninivite, plus complète. La seconde est postérieure à la première, qui remonte aux xvIII et xvII ${ }^{\mathrm{e}}$ siècles avant Jésus-Christ, et les fragments qui nous en sont parvenus s'échelonnent sur une longue période. C'est celle que nous lisons habituellement aujourd'hui. Cela étant, si le paysage dans lequel Danis fait évoluer ses Azzédiens offre quelque parenté avec celui où évolue le roi d’Uruk, au total, les différences entre l'épopée et le drame sont bien plus frappantes que les ressemblances. Une autre exception subsiste cependant: l'immortalité scellée par l'écriture que leur quête vaut aux deux héros. Mais Gilgameš n'est divinisé qu'après coup, hors du récit, alors que la sainteté est conférée à J'il à la toute fin d'e. Roman-dit. Quoi qu'il en soit, seule une étude comparative permettrait de traiter cette question dans toute son ampleur. Pour plus de détails sur la naissance du poème épique, voir L'épopée de Gilgameš. Le grand homme qui ne voulait pas mourir, traduit de l'akkadien et présenté par Jean Bottéro, Paris, Gallimard, coll. «L'aube des peuples», 1992, p. 17-59.

5 Autre procédé courant chez Danis: celui de fabriquer des mythes à partir d'événements historiques et de problèmes plus ou moins contemporains. Pour ce faire, il poétise ces événements et ces problèmes et les détache de l'aire géographique et du cadre temporel dans lesquels ils se situent pour les présenter comme une réponse générale à une question plus vaste. Par exemple, e. Roman-dit fait allusion au problème des réfugiés et à celui des Métis, tandis que Le pont de pierres et la peau d'images renvoie à l'épisode des «boat people» et au travail des enfants. Daniel Danis, Le pont de pierres et la peau d'images, Paris, L'École des loisirs, coll. «Théâtre», 1996, 94 p. Désormais, les références à cet ouvrage seront indiquées par le sigle $P P$ suivi du folio, et placées entre parenthèses dans le texte. 
joue son royaume aux dés, alors que J'il y apprend que Dadagobert a perdu la terre de Tableau au cours d'une partie de cartes avec Blackburn (E, 60). Auparavant, au «Tableau noir de craie», J'il est clairement décrit comme un Métis pourvu de tresses - en quoi il est associé à la tradition autochtone -, tresses qu'il doit se résoudre à couper contre sa volonté $(E, 39)$. Le dernier tableau a, quant à lui, des résonances bibliques. Pareil à Moïse qui meurt sans avoir atteint la Terre promise, J'il y déclare solennellement avant de s'éteindre: «Nous marchons vers une terre d'accueil qui est encore à cent heures de paroles d'ici, frère-homme.» $(E, 118)$

Première observation: Danis réinvente le matériau mythique dans une optique de patchwork. Pareilles aux couvertures fabriquées de cette façon, ses pièces acquièrent relief et couleurs en raison de la profusion baroque - d'autres la qualifieraient sans doute de postmoderne - que l'auteur dramatique obtient en cousant ensemble des carreaux de surnaturel de provenances variées. Bien que ses drames puissent être logés à l'enseigne du patchwork d'un point de vue mythocritique, leur unité ne leur vient pas de ce niveau microscopique. Celle-ci se situe davantage, me semble-t-il, au niveau macroscopique: par les mythes auxquels fait appel l'auteur, lesquels montrent une certaine parenté les uns avec les autres ${ }^{6}$. Mais vers quels types de mythes Daniel Danis se tourne-t-il le plus souvent?

\section{MYTHES DE CRÉATION ET MYTHES DE DESTRUCTION}

Avant de répondre à cette question, précisons d'abord le terrain conceptuel sur lequel nous nous appuyons. L'idée de classer les mythes n'est pas nouvelle. Elle peut mener, bien entendu, à une trop grande simplification. Au dire de Jean-Paul Giraud, toutefois, c'est une étape nécessaire pour le chercheur qui veut les étudier afin, surtout, «d'éviter les écueils qu'un comparatisme trop hâtif [...] risquerait d'engendrer ${ }^{7}$ ». Si Giraud suggère en premier lieu de les classifier en récits purement oraux, transcrits par des scribes, ou en récits à proprement parler littéraires, son deuxième critère m'apparaît plus pertinent encore, à savoir non pas essayer de dater les mythes en fonction de l'époque où ils sont apparus, mais plutôt par la proximité qu'ils entretiennent, au sein de la cosmogonie dont ils font partie, avec le récit d'explication de l'origine du cosmos auquel ils se rattachent. Giraud propose ainsi de distinguer les mythes en récits cosmogoniques et en récits de création. On peut ensuite subdiviser ces derniers en fonction des créatures et des éléments auxquels ils donnent

6 Il est important de signaler que Danis emprunte moins à ces grands récits des fables et des héros spécifiques que le caractère légendaire d'au moins un personnage et l'ampleur d'une catastrophe ou d'une apothéose qui reposent rarement sur des causes naturelles. Ceci m’amène à préciser la manière dont je considérerai le mythe dans ce texte. Deux conditions définitoires retiendront avant tout mon attention. Premièrement, «le mythe est un ensemble qui ne saurait se réduire à une situation simple». Deuxièmement, le mythe convoque un mystère dont le commun des mortels ne saisit pas la cause et formule une explication à son sujet sans le secours de la raison ni de l'expérience scientifique. Dans cette perspective, les mythes génésiques constituent la forme idéale du mythe. Voir à ce sujet Pierre Brunel, Mythocritique. Théorie et parcours, Paris, Presses universitaires de France, coll. «Écriture», 1992, p. 18-29.

7 Jean-Paul Giraud, «Typologies des mythes», Daniēle Chauvin, Andrê Siganos et Philippe Walter (dir.), Questions de mythocritique. Dictionnaire, Paris, Imago, 2005, p. 359. 
naissance ${ }^{8}$. Le récit cosmogonique, qui met de l'ordre dans le cosmos (souvent en dissociant la Terre du Ciel), précède généralement les mythes de création. Ces derniers permettent ensuite aux divers éléments, créatures, communautés, objets d'apparaître et de mettre ce monde en mouvement, de l'animer, en quelque sorte, par les multiples activités qui rythment la vie humaine. À l'autre bout du spectre apparaissent les mythes de destruction, en d'autres mots, les eschatologies ou les récits apocalyptiques, «qui semble[nt] véhiculer l'idée du mythe de la Fin ${ }^{9}$ » avec un grand «F». Jean-Paul Giraud observe cependant que «la plupart de ces récits augurent d'un anéantissement, mais avec la présupposition d'une nouvelle création, cette fois-ci, parfaite $^{10} »$. Il est donc clair que les mythes de destruction et de création sont intimement liés, puisque les uns préparent les autres.

Un tel enchaînement est très présent dans le théâtre de Danis, où le récit de destruction s'accompagne souvent d'une nouvelle création ou, tout au moins, d'une renaissance quelconque. Dans ses pièces, mythes de destruction et de création vont souvent de pair, tout comme sont unis les mythes et les rituels. Au reste, Manilowski a démontré que l'interdépendance des mythes et des rituels est complexe, alors que l'anthropologue américain Clyde Kluckhohn attribue aux deux entités une fonction commune: celle de réduire le niveau d'anxiété dans la population ${ }^{11}$. Tel semble aussi être le cas chez l'auteur de Cendres de cailloux ${ }^{12}$, où rituels et mythes se renforcent les uns les autres et interviennent à des moments cruciaux de l'intrigue.

Pour illustrer cette cohabitation des mythes de destruction et de création dans le théâtre de Daniel Danis, mais aussi celle de mythes et de rituels, tournonsnous vers une pièce peu connue destinée au jeune public: Le pont de pierres et la peau d'images. Deux enfants de deux régions différentes - on le sait parce qu'ils ne parlent pas la même langue - fuient la guerre afin de pouvoir «vivre la paix»

8 «Les mythes de création ou d'origine sont donc la réitération narrative, parfois allégorique, des actes divins nécessaires à l'ensemble des créations qui vont servir à consolider et à dynamiser l'univers précédemment créé mais, aussi et parfois, à l'appauvrir ou à en limiter la dimension socio-cosmique. De facto, l'émergence, [dans un ordre donné] des minéraux, des végétaux, des formes animales, des divers êtres surnaturels, des hommes, des éléments climatiques, du feu, de l'agriculture, des concepts sociaux, des interdits, des tabous, des institutions, des rites, etc., s'inscrit parfaitement dans le cadre de ces récits au même titre que l'origine de la mort, de la maladie, des enfers, des calamités, des fléaux...» Ibid., p. 363-364.

9 Ibid., p. 367.

10 Ibid.

11 "In short, the only uniformity which can be posited is that there is a strong tendency for some sort of interrelationship between myth and ceremony and that this interrelationship is dependant upon what appears, so far as present information goes, to be an invariant function of both myth and ritual: the gratification (most often in the negative form of anxiety reduction) of a large proportion of individuals in a society. " Clyde Kluckhohn, «Myths and Rituals: A General Theory», John B. Vickery (dir.), Myth and Literature. Contemporary Theory and Practice, Lincoln, University of Nebraska Press, 1969, p. 37. «En somme, la seule uniformité que l'on puisse observer entre le mythe et la cérémonie est qu'ils ont une forte tendance à former une sorte d'interrelation et que cette interrelation relève de ce qui semble être, pour ce que nous en savons aujourd'hui, une fonction permanente à la fois du mythe et du rituel, soit la satisfaction du désir (le plus souvent sous la forme négative d'une réduction de l'anxiété) d'une large partie de la population d'une société. » Je traduis.

12 Daniel Danis, Cendres de cailloux, Montréal/Paris, Leméac/Actes Sud-Papiers, 2000 [1992], 122 p. Désormais, les références à cet ouvrage seront indiquées par le sigle CC suivi du folio, et placées entre parenthèses dans le texte. 
$(P P, 17)$. Ces enfants seront, au contraire, réduits en esclavage dans une usine de tapis et connaîtront bien d'autres péripéties avant de trouver refuge dans un nouveau pays qu'ils recréent de toutes pièces.

Le récit de destruction est particulièrement dur, et ce, même s'il est destiné au jeune public, puisque non seulement le pays est détruit, mais cette destruction est suivie d'une dislocation de la famille et d'une mise en esclavage des enfants par un passeur sans scrupules. Les enfants ne réussissent ensuite à gagner «le Pays sans Guerre» $(P P, 22)$ qu'en montant, au péril de leur vie, dans des «embarcations de fortune» $(P P, 84)$. Scène apocalyptique que cette ultime pérégrination décrite ainsi par les héros de la pièce, la petite Mung et le petit Momo:

\author{
MUNG \\ Des barques et des barques remplies de jeunes \\ comme nous deux \\ Certains étaient assis depuis si longtemps \\ dans le fond des embarcations \\ que leurs fesses de culotte avaient gelé tant \\ qu'elles étaient collées sur le bois. \\ MOMO \\ Et maigres, maigres de malheur. \\ Creux, creux, les yeux. \\ MUNG \\ Et si le vent se levait d'une grande force \\ il les casserait comme une vitre. $(P P, 84-85)$
}

À la destruction succède bientôt la création, aussitôt que les enfants abordent la Terre promise. Voici un extrait du miracle qui s'y produit:

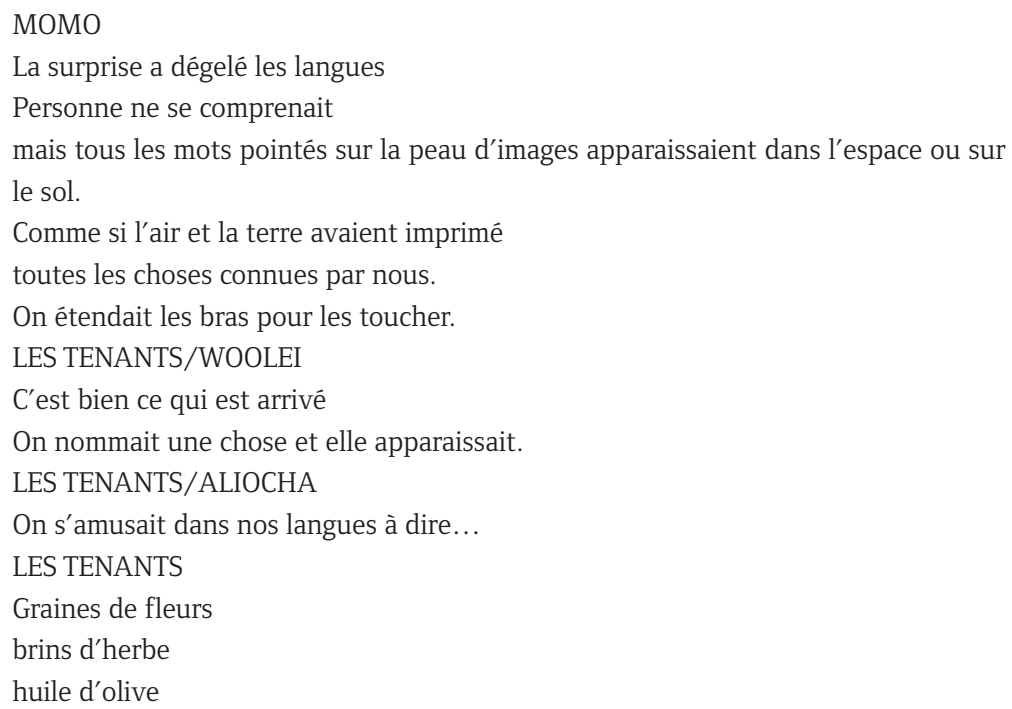


tiges de fer

roues de pierre

papier de coton. $(P P, 88-89)$

Le récit de création se poursuit encore sur plusieurs paragraphes. Mais il s'accompagne surtout de deux rituels. L'un est lié à la parole et passe par une activation de la peau d'images confiée à Mung par ses parents; l'autre relève du monde concret et provient du vœu émis par la mère de Momo que son fils suive la tradition familiale et se fasse à son tour bâtisseur de ponts de pierres.

La destruction et la création d'un monde nouveau se déclinent de manière similaire dans e. Roman-dit, qui raconte l'épopée de J'il et des Azzédiens. Cette épopée s'ouvre sur des images de destruction elles aussi causées par la guerre, images que l'auteur met dans la bouche d'une jeune fille: «Dis-moi, les images de ma courte vie vont-elles s'évanouir ou résister à nos maisons éventrées, à nos ustensiles de cuisine éparpillés, à notre ville renversée comme un verre de lait sur une plaque chauffante?» $(E, 15)$ Une fois de plus, la destruction est suivie d'un long exil, d'un interdit de construire des habitations permanentes et même d'une autre guerre pour les Azzédiens, avant qu'ils ne partent à la recherche d'un monde de paix. Le projet sera rendu possible par la mort du héros qui, peu avant sa disparition, invite les siens à mettre en œuvre un monde de paix en souvenir de lui: «Habitez-moi dans l'allégresse. Peuplez-moi d'un monde infini de paix.» $(E, 118)$ Aux mythes de destruction et de refondation du monde sont aussi associés des rituels. Le principal réside dans les écritures inspirées de la vie de J'il qui sont censées rappeler son enseignement et qui se retrouvent mises en abyme dans la pièce. Un peu plus et on croirait entendre le héros messianique dire: «Faites la paix en mémoire de moi.»

D'autres pièces de Danis sont davantage centrées sur un seul motif, soit celui de la destruction, soit celui de la création, parfois sous la forme d'une renaissance individuelle. En outre, quelques pièces proposent aussi des tentatives de recréation du monde qui échouent. Le langue-à-langue des chiens de roche présente ce topos: la tentative de rebâtir un paradis terrestre sur une île fictive du Saint-Laurent est vouée à l'échec en dépit des efforts de deux Amérindiennes violées puis chassées de leur réserve, Joëlle et Déesse, qui voulaient y refaire leur vie. À la fin, la catastrophe survient tout de même. Déesse le déclare sans ambages: "L'île a chaviré ${ }^{13}$.» Toutefois, Niki et Djoukie, la fille de Joëlle, ne sont pas morts en vain, puisque leur cri «Au secours d'amour!», en faveur d'une humanité «réhumanisée», a pu retentir. Cet échec s'explique-t-il par l'état de délabrement physique et psychologique avancé des êtres qui peuplent l'île? L'animalité qui gouverne la plupart d'entre eux peut le laisser entendre. Toujours est-il que les habitants de l'île n'arrivent pas à retrouver l'élan vital nécessaire pour recréer la vie. Simon, le nouveau venu, en est un bon exemple. De retour de Bosnie, le soldat traumatisé a beau déclarer que «[d]eux corps qui s'unissent pour refaire la vie, ça devrait suffire» $(P P, 58)$, il n'en continue pas moins d'être poursuivi par des images de guerre. C'est aussi compter sans le passé criminel de son ami Charles - lequel n'a pas sauvé de la noyade un vendeur de drogue

13 Daniel Danis, Le langue-à-langue des chiens de roche, Montréal, Leméac, 2007, p. 76. 
qui voulait l'escroquer. Les amis de ce dealer, forces maléfiques qui empêcheront les rituels «rage ${ }^{14}$ » de Coyote de faire renaître la vie dans cette île, se vengeront ensuite à plusieurs reprises sur son plus jeune frère, Niki. Comme dans Roméo et Juliette, la jeunesse est sacrifiée en vain dans ce drame et, avec elle, l'espoir de ramener à la vie les héroïnes et les proches touchés par la violence qu'elles ont subie.

Le topos du retour à la vie contrarié apparaît dans Cendres de cailloux et dans Celle-lâa ${ }^{15}$, dont les personnages principaux essaient de recommencer à zéro ${ }^{16}$ en allant symboliquement habiter une nouvelle maison. La violence que cherchent à fuir la Mère de Celle-là et le Clermont de Cendres de cailloux se déchaîne cependant à nouveau sur eux sans qu'ils puissent l'arrêter. Terre océane dépeint, au contraire, le retour à la vie réussi d'Antoine, qui est rendu possible parce qu'il accepte d'accompagner vers la mort Gabriel, son fils adoptif, non sans aller chercher, pour y parvenir, l'aide de son "chaman ${ }^{17}$ » d'oncle et de ses rituels. Ce pacte qu'Antoine conclut implicitement en décidant d'héberger ce fils expédié sans avis par son "ex", incapable de composer avec le cancer dont le garçonnet est atteint, oblige le héros à se reconnecter avec la «vraie vie», qu'il avait délaissée au profit de la «cinémenterie ${ }^{18}$ ».

\section{L'ANCRAGE MAGIQUE}

Terre océane repose donc sur un «fonds de magie diffuse ${ }^{19}$ » qui s'y manifeste par la présence d'un chaman et par la notion du pacte ${ }^{20}$, qui paraît aussi relever de la mentalité préscientifique à laquelle adhèrent plusieurs personnages inventés par Danis. Mais c'est loin d'être la seule pièce qui baigne dans cette ambiance. Au total, rarement a-t-on vu œuvre dramatique réunir autant d'êtres qui détiennent ou, du moins, sont soupçonnés de détenir des pouvoirs magiques ou d'avoir maille à partir avec le surnaturel. La Mère de Celle-là n'est que la première figure du long cortège de sorcières, de magiciens, de chamans et de prophétesses qui peuplent cet œuvre dramatique. En effet, il n'est pas une pièce de Danis où l'on ne retrouve au moins un être doté de «mana», pour reprendre le mot par lequel Marcel Mauss désigne, dans son «Esquisse d'une théorie générale de la magie», la force spirituelle - bénéfique ou maléfique - qui émane du magicien ${ }^{21}$.

14 Jeu de mots de Daniel Danis inventé à partir des partys rave.

15 Daniel Danis, Celle-là, Montréal, Leméac, 1993, 91 p. Désormais, les références à cet ouvrage seront indiquées par le sigle $C L$ suivi du folio, et placées entre parenthèses dans le texte.

16 Dans le carnet de Clermont, il est écrit, selon sa fille Pascale, qui en fait la lecture: «Recommencement. An zéro.» (CC, 24)

17 Daniel Danis, Terre océane, Paris, L'Arche, 2006, p. 44.

18 Ibid., p. 40. C'est ainsi que Danis fait désigner le cinéma dans cette pièce par l'oncle d'Antoine. Il faut savoir que ce dernier est producteur de cinéma et que son métier est justement ce qui l'éloigne d'une vie plus en phase avec la nature.

19 Marcel Mauss, «Esquisse d'une théorie générale de la magie», L'Année sociologique, 1902-1903, p. 87.

20 Mauss, qui nous sert de guide dans la présente partie, souligne que le magicien et les démons sont souvent liés aux «thèmes du jeu, du pacte et de la descendance». Ibid., p. 24.

21 Pour Mauss, le mana est une force spirituelle propre au magicien. C'est dans la mesure où le magicien a du mana qu'il peut agir sur les esprits, les évoquer, les commander et les posséder. Ibid., p. 70. 
Campée dans la Grande Noirceur des années 1950 au Québec, l'héroïne de Celle-là est considérée comme une "vraie sorcière ${ }^{22}$ » par son entourage. Qu' est-ce qui lui vaut d'être traitée ainsi? L'épilepsie dont elle est atteinte. Pour ses congénères, cette maladie est le signe de sa possession par le démon ${ }^{23}$. Cette explication préscientifique est confirmée autour d'elle par le comportement sexuel débridé dont elle fait preuve, puis par les blessures qu'elle inflige à son fils au cours d'une crise qui la surprend ciseaux en main. Élevée dans la foi chrétienne et habituée à porter les stigmates de l'exclusion, la jeune femme se perçoit elle-même comme possédée en dépit des explications fournies par le médecin sur son mal:

\author{
Oui, c'est revenu. \\ Le médecin m'a dit aussi: \\ «Quand le sang coagule \\ une sorte d'épilepsie \\ le cerveau, il bloque.» \\ Là, j'tombe par terre. \\ Le corps se creuse un trou dans le plancher. \\ Une sorte de magie que j'ai. \\ Une sorcière, hein! Tu penses. \\ Ça, c'est les pilules contre le creusage de trous. (CL, 14)
}

En fait, il serait plus juste encore de dire que la mentalité scientifique et la pensée magique cohabitent à l'époque, mais que la pensée magique finit par s'imposer dans la mesure où elle offre un meilleur cadre explicatif aux nombreux interdits religieux qui pèsent alors sur la société québécoise ${ }^{24}$. Or quels sont les pouvoirs magiques qui portent les gens à assimiler l'héroïne à une sorcière? Érotisme et violence. Son pouvoir de création ${ }^{25} l^{\prime}$ amènera à concevoir un enfant hors des liens du mariage (avec le Vieux), tandis que son pouvoir de destruction s'exercera sur le bambin... qu'elle percera de coups de ciseaux! En guise de châtiment, non seulement la Mère en serat-elle séparée à jamais, mais elle sera assassinée dans son antre par «[u]ne bande de jeunes» (CL, 5). Tel est le sort cruel réservé aux sorcières dans le Québec obscurantiste évoqué par Danis.

Dans Cendres de cailloux et Le chant du Dire-Dire ${ }^{26}$, l'influence du magique et du surnaturel s'accroît. En effet, les deux drames présentent davantage encore

22 «C't'une vraie sorcière.» $(C L, 12)$ Tel est le cri lancé à la Mère par un des enfants venus frapper à sa porte un soir d'Halloween. Cela étant, le sentiment est partagé non seulement par le village, mais par sa famille, à l'exception notable de son frère qui deviendra évêque, seul d'entre les siens à lui venir en aide au cours de la pièce.

23 "Après une réunion familiale/on a rendu la sentence/quitter la maison paternelle/“A fait ses crises de démone. Occupe-toi-z'en, j’suis pus capable d'la voir." /Ma mère criait ça à mon frère savant.» (CL, 24-25)

24 «La magie, indique Marcel Mauss, a une véritable prédilection pour les choses interdites. » «Esquisse d'une théorie générale de la magie», p. 81.

25 «LA MÈRE: Ma vie s'est arrêtée./J'avais vingt-huit ans./Pour la première fois de ma vie/j'avais créé quelque chose./Avec le plaisir de mon corps./Ça m'est plus jamais arrivé/de réussir à créer.» $(C L, 28)$

26 Daniel Danis, Le chant du Dire-Dire, Paris, Théâtre Ouvert, coll. «Tapuscrit», 1996, 88 p. 
d'agents magiques que Celle-là. On peut même y distinguer des confréries ou des fratries $^{27}$. Confréries au sein desquelles la figure féminine centrale de la pièce joue un rôle déterminant. Le départ définitif de Shirley dans la première et celui, périodique, de Noéma dans la seconde mettent indéniablement le groupe à rude épreuve. En outre, si les deux confréries ont partie liée avec le surnaturel et vivent à l'écart du monde civilisé ${ }^{28}$, elles sont de nature différente. L'une est qualifiée par l'un des frères (Fred-Gilles) de Société d'Amour Durant, c'est-à-dire qu'elle est vouée au bonheur de ses membres, tandis que l'autre, constituée de Shirley et des quatre "p'tits gars» $(C C, 10)$, s'adonne à «des rituels macabres ${ }^{29}$ » et tend en d'autres mots vers le maléfice. Le signe distinctif de Shirley - le mot «Macchabée» tatoué par son père sur l'un de ses seins, alors qu'elle avait seulement trois ans - en est une preuve supplémentaire, la liant au culte des morts, tandis que le talent de Noéma pour le chant et l'accordéon qui apparaît au moment où le Tonnerre foudroie ses parents en fait une "sirène des naufragés ${ }^{30}$ ». Associée aux éclairs et au ciel, cette dernière paraît être une incarnation du divin. Son élan vital ne manque pas de se tarir au contact du public «des bars dans toutes les contrées ${ }^{31} »$ pour lequel elle chante ${ }^{32}$. Sa nature divine n'échappe pas non plus aux "municipiens ${ }^{33}$ ", qui s'arrachent ses reliques.

Il n'en est pas de même avec Shirley, qui, très tôt, se montre consciente des pulsions mauvaises qui l'habitent et affirme: «Je possède un pouvoir de destruction.» (CC, 23) Elle aimerait néanmoins troquer cette faculté de causer la destruction contre celle de créer la vie: "Avoir une serre. Faire pousser les fleurs. Le commerce des odeurs.» $(C C, 68)$ La rencontre de Clermont, de qui elle tombe amoureuse, lui fait croire qu'elle pourra y parvenir. Elle va même jusqu'à lui remettre un talisman destiné à le protéger. C'est compter sans Coco, qui ne cesse d'enjoindre à Shirley de tenir le serment de «faire la fête à Caillou» — surnom de Clermont, serment qu'elle a prononcé un soir par bravade ${ }^{34}$. Il espère ainsi la détacher de Clermont, la garder auprès de lui et conserver intacte la confrérie. En fait, au fur et à mesure que la faim d'annihiler décroît chez Shirley, elle croît chez Coco. L'esprit d'un animal dénommé Gulka ${ }^{35}$ s'empare peu

27 Dans Le chant du Dire-Dire, les Durant L'Orage constituent une famille entretenant des liens étranges avec le surnaturel, comme l'indique son surnom. De son côté, le quintette de Cendres de cailloux arbore des traits caractéristiques des sociétés secrètes, telle la prédilection de ses membres pour la transe, le désordre et les rites reliés à la mort, explicitée dès le premier tableau: «SHIRLEY: On ne peut pas déterrer quelqu'un/ même mort depuis seulement six jours/sans se soûler la gueule./On tombait en transe/quand on faisait des affaires/qui sortaient du bon sens./"Dansez, dansez, les petits gars." /On était cinq/toujours les mêmes cinq./Flagos, Dédé, Grenouille, moi/pis celui qui danse avec la chienne à Thibodeau:/Coco.» (CC, 9) Outre cette confrérie, Shirley consulte un couple de magiciens formé d'un voyant qui lit dans les feuilles du thé et d'une «ramancheuse» : monsieur et madame Huot.

28 Selon Mauss, c'est l'une des conditions de la magie que de se produire dans des lieux à l'écart.

29 Marie-Christine Lesage, «Archipels de mémoire. L'œuvre de Daniel Danis», p. 81.

30 Daniel Danis, Le chant du Dire-Dire, Montréal, Leméac, 2006, p. 22.

31 Ibid.

32 L'épuisement de Noéma est attribué par Rock à l'alcool que des hommes lui ont fait boire: «Je vais les tuer pour avoir grisé notre sœur de même!»Ibid., p. 30.

33 Ibid., p. 18.

34 On l'a vu, les serments, les pactes et les jeux dangereux sont monnaie courante dans les sociétés secrètes.

35 Gilbert David est d'avis que l'emploi du néologisme «Gulka» pour désigner l'animal qui a mordu le cerveau de Coco est calqué «sur le modèle des Pégase, Sphynx ou Griphon, tous animaux fabuleux» («Le langueà-langue de Daniel Danis», p. 75). 
à peu du jeune homme, réveillant la violence qui gronde en lui. Là encore, cet esprit malin s'est insinué en lui peu après sa naissance ${ }^{36}$. Et l'animal n'aura de cesse qu'après avoir anéanti celui qu'il hante ${ }^{37}$ : renié par Shirley à la face du monde, Coco se loge une balle dans la tête.

En gros, les deux pièces semblent suivre un mouvement inverse. Tarissement du pouvoir de création exprimé par le chant et l'exaltation du verbe dans Le chant du Dire-Dire, qui conduit pratiquement à l'autodestruction des frères et à l'assomption de Noéma, soulevée hors de l'eau par son mari Tonnerre. Anéantissement du pouvoir de destruction et dislocation du groupe qui l'incarne dans Cendres de cailloux, culminant par la mort de Coco et la folie de Clermont, mais aussi - on a tendance à l'oublier - par la profession de foi en la vie de Shirley. Elle l'exprime à deux reprises. Au salon funéraire, elle lance à Jacques (le vrai prénom de Coco): «Jamais, jamais, tu m'entends, je n'arrêterai de vivre. [...] J'vas continuer de parler à la terre/de faire pousser des fleurs.» (CC, 120) Elle promet en outre à Clermont le recommencement dont il rêvait:
Je te redonnerai une langue.
On fera des choses que personne ne fait.
Le soir, on fera monter la lune avec un soufflet.
Au matin, on fera lever le soleil avec des poulies
qu'on accrochera aux arbres de notre forêt.
On tirera fort pour lever le soleil.
Je te ferai sortir des enfants de mon ventre.
On les élèvera dans une échelle de bois. (CC, 117)

On remarquera dans tous les cas l'accent mis sur la langue, sur la puissance du verbe et sur la faculté qu'ont les mots d'évoquer la recréation du monde ou d'exorciser sa destruction en des paroles proches de l'incantation, parfois accompagnées de gestes rituels, l'un renforçant l'autre. Célébration, au fond, du potentiel magique des mots qui ont, dans ces poèmes dramatiques, le pouvoir de faire apparaître et disparaître les choses et amènent les êtres, même par-delà la mort, à raconter l'emprise exercée sur eux par Éros et Thanatos.

En fait, les rites, qu'ils soient oraux ou gestuels, paraissent constituer dans ce thêâtre le lien qui fédère le substrat magique ainsi que les références et les structures mythiques inhérents à cet imaginaire singulier. Il n'est sans doute pas innocent que des rituels aux sonorités étranges donnent leur nom à tant de pièces de Danis: langue-à-langue, chant du Dire-Dire, construction de ponts de pierres, consultation de la peau d'images, récitation/écriture d'un roman-dit, plongée dans la terre océane. La plupart ont partie liée avec la pulsion de vie, l'instinct de survie ou le désir de prolonger l'expérience humaine au-delà de la mort. Il n'en résulte pas pour autant une

36 «COCO : Dans mon crâne se promenait un tout petit animal de ma grandeur qui hurlait de faim. C'est à partir du lait de ma mère qu'il a commencé à grandir.» (CC, 32)

37 Selon Mauss, le dédoublement sous l'aspect animal caractérise souvent le sorcier et indique que le pouvoir du magicien a son origine en dehors de lui-même («Esquisse d'une théorie générale de la magie», p. 22-23). 
vision apaisante de la condition humaine. Au contraire, Danis dépeint constamment l'être humain comme vivant dans un monde violent, chaotique, aliénant, qu'il doit fuir ou encore chercher à réinventer, sans toujours y parvenir tant sont nombreux les obstacles intérieurs et extérieurs qui s'opposent à lui. Danis jette ainsi une passerelle invisible entre ce qui perdure, selon lui, d'hier à aujourd'hui en mettant au jour la difficulté des êtres humains à s'extirper du cercle de la violence et du chaos primitif auquel nous sommes à tout moment menacés de retourner.

Au terme de cette lecture de l'aura mythique du thêâtre de Danis, nous tombons d'accord avec l'auteur dramatique lorsqu'il affirme que « [s] on écriture est ancrée dans ce mouvement fondamental de recréation et de destruction ${ }^{38}$ ». Les mythes de la destruction ou de la création qu'il convoque déterminent notamment les deux tonalités qui dominent dans son thêâtre, si on suit les archétypes que Northrop Frye associe à ces mythes ${ }^{39}$. Selon Frye, la création, la renaissance et le retour à la vie du héros sont liés au printemps. Le mode d'expression privilégié des mythes printaniers est l'épopée ou la poésie rhapsodique. En revanche, Frye estime que la destruction et la mort sont plutôt des mythes associés à l'automne. On retrouve souvent dans ceuxci les personnages du traître et de la sirène. L'élégie et la tragédie sont les formes les mieux à même d'exprimer ces mythes. Or quelle combinaison trouvons-nous dans la poétique de ce thêâtre qui traite sans cesse de création et de destruction? L'épique et le tragique qui se donnent la main pour renouer avec ce que j'appelais ailleurs les «éternels mystères de la vie et de la mort ${ }^{40}$ ». Encore que l'épique y prédomine sans contredit ${ }^{41}$, indiquant, à mon sens, l'intention de Danis de produire un récit doté de résonances collectives.

Reste à se demander pourquoi l'auteur insiste à ce point sur ces deux dimensions de la vie collective que sont la création et la destruction, outre qu'elles sont constitutives de l'expérience humaine. Marie-Christine Lesage y répond en quelque sorte lorsqu'elle estime que Danis s'intéresse au problème de l'après-catastrophe. En d'autres mots, écrit-elle, "comment exister, vivre, aimer, jouir avec tant de cendres derrière soi et avec la conscience aigüe de la mort à ses côtés ${ }^{42}$ ?». Une autre interprétation nous est fournie par la principale utopie véhiculée par Danis à l'aide de ces mythes: essentiellement, l'urgence d'instaurer une Terre sans guerre, un monde dans lequel, à tout le moins, la violence ne serait pas aussi présente. Utopie d'une humanité débarrassée de sa barbarie ${ }^{43}$, au sein de laquelle l'amour fusionnel et le dialogue - le langue-à-langue entre êtres humains en chair et en os - seraient moins souvent contrecarrés. Naturellement, Daniel Danis sait mieux que quiconque que le paradis sur Terre ne dure jamais longtemps.

38 Luc Boulanger, «Le langue-à-langue des chiens de roche», p. 49.

39 Les réflexions de Northrop Frye proviennent de son article «The Archetypes of Literature», John B. Vickery (dir.), Myth and Literature, p. 87-97.

40 Hervé Guay, «Le retour à la terre», Le Devoir, 29 octobre 2007, p. B2.

41 Voir à ce sujet l'excellente étude de Nadine Desrochers, "Le récit dans le théâtre de Daniel Danis», p. 119-132.

42 Marie-Christine Lesage, «Archipels de mémoire. L'œuvre de Daniel Danis», p. 83.

43 Dans son étude sur Cendres de cailloux, Christian Saint-Pierre en vient à une conclusion similaire: «Dans cette danse de la vie et de la mort, n'y a-t-il pas en fin de compte un appel à briser le cycle de la violence?» Christian Saint-Pierre, «Quand la mort danse avec la vie. Cendres de cailloux de Daniel Danis», p. 67. 